\title{
Principes, méthodes de la recherche en partenariat : une proposition pour la traction animale
}

\author{
E. Chia ${ }^{1}$
}

\section{Mots-clés}

Energie animale - Recherche Partenariat - Innovation - Gestion Afrique au sud du Sahara.

\begin{abstract}
Résumé
La rencontre des chercheurs qui s'interrogent sur l'efficacité de leurs interventions pour accompagner les acteurs dans les processus de changement constitue une occasion pour s'interroger sur les méthodes de recherche à développer lors de travaux réalisés avec les acteurs : recherche participative, « recherche-action», recherche intervention... L'auteur propose de présenter la démarche de recherche-action comme nouvelle. Si les trois phases de la recherche-action sont importantes, la phase de négociation est capitale car elle permet la formalisation du contrat entre les partenaires (cadre éthique), la fabrication d'un langage commun, et la formalisation d'une problématique structurante entre chercheurs de disciplines différentes et acteurs. Dans la démarche de recherche-action, des dispositifs de gouvernance (comité scientifique...) jouent un rôle très important: ils sont à la fois garants de la résolution des problèmes et de la production, et de la légitimation des connaissances scientifiques produites. En conclusion sont proposées des pistes pour construire des recherches-action dans le domaine de la traction animale, afin d'imaginer de nouvelles innovations sociotechniques et organisationnelles pour faciliter l'utilisation de cette technique.
\end{abstract}

\section{INTRODUCTION}

Depuis le début des années 1990, les Etats africains, sous la pression du FMI et de la Banque mondiale, ont commencé à transférer au secteur privé et aux collectivités territoriales un certain nombre d'activités qu'ils avaient jusque-là assurées *. C'est le cas en particulier des services, comme le crédit, la vulgarisation et le conseil aux exploitations agricoles.

Dans ce contexte, de libéralisation et de décentralisation, certains agriculteurs des régions productrices de coton, d'arachide, de café, appuyés par des bailleurs de fond, se structurent en organisations professionnelles (OP) ou interprofessions. Dans les zones dépourvues de cultures commerciales, ce mouvement est plus lent, voire inexistant.

1. Inra-UMR Innovation / Cirad - Ideas, 73 avenue J.-F. Breton,

34398 Montpellier Cedex 5, France

E-mail : chia@ensam.inrafr
Le modèle de vulgarisation descendant, jusqu'ici utilisé pour proposer aux agriculteurs des changements, donne de moins en moins de résultats, car il ne permet pas de prendre en compte les activités et les besoins des agriculteurs ainsi que les contextes d'action. Cependant, travailler avec les acteurs, pour identifier leurs besoins et coproduire, coconcevoir les changements ou les innovations, n'est pas une tâche aisée $(2,7,8)$. Il faut changer les dispositifs et les pratiques de la recherche : passer d'une recherche disciplinaire à une recherche pluridisciplinaire, d'une recherche en laboratoire à une recherche avec les acteurs, et faire face non seulement aux incertitudes techniques et scientifiques mais également aux incertitudes sociales.

$\mathrm{La}$ « recherche-action » (RA) est non seulement une façon de prendre en compte les situations et les besoins des agriculteurs, mais

* Un paysan du Burkina Faso a dit à l'auteur, en parlant du désengagement de l'Etat : «Qui est l'Etat ? Est-ce que l'Etat est vraiment intervenu pour nous aider ?... » Ceci traduit le fait que, pour une majorité d'agriculteurs africains, en réalité l'Etat ne s'est jamais engagé à leurs côtés. Pour se désengager, encore faut-il avoir été engagé ! 
aussi un vecteur du développement et du changement. Dans le cas du développement de la traction animale, le désengagement des Etats s'est traduit par le renforcement du rôle des forgerons et la faillite des usines d'agroéquipements, par le transfert des services aux OP (d'appui/conseil) et au secteur privé (marchés financiers, vétérinaires mandataires...). De ce fait, de nouvelles relations de service et de conseil voient le jour. Elles sont à la fois le produit du développement de la traction animale et le contexte de son développement - ou de son non-développement. Ces nouvelles relations, de type coopératif ou conflictuel, qui s'établissent entre les différents acteurs conditionnent les processus de coconception des innovations sociotechniques et organisationnelles au niveau local.

Ces mutations conduisent la recherche agronomique à s'interroger à la fois sur les contours et la nature de l'objet de recherche (configurations d'acteurs...), et la manière de conduire des recherches avec l'ensemble des acteurs concernés pour concevoir avec eux des innovations sociotechniques et organisationnelles.

Afin de contribuer à répondre à cette problématique, dans une première partie seront présentées les principales caractéristiques de l'innovation en agriculture, et des relations qui s'établissent entre les agriculteurs et les conseillers (ou chercheurs), lors d'une situation de conseil. Dans une deuxième partie, l'auteur illustrera, en s'appuyant sur deux expériences, une démarche de recherche en partenariat (20), la RA, qui favorise le travail de coconception des innovations ou d'élaboration des innovations en partenariat. En conclusion, sera faite une proposition d'organisation d'une recherche en partenariat dans le cas de la traction animale.

\section{- INNOVATION ET RELATIONS DE CONSEIL EN AGRICULTURE}

L'innovation (11) est un processus incertain et complexe $(3,4)$ qui comprend des phases de planification et des phases d'opportunité L'innovation ne s'adopte pas, elle se crée et se construit socialement. Elle est le produit de l'interaction des acteurs et en particulier des agriculteurs et conseillers qui vont créer le cadre sociotechnique (les règles, les interrelations, les dispositifs) au sein duquel les nouvelles techniques ou inventions vont fonctionner (25). La conception de l'innovation se fait donc en partenariat. Le partenariat est défini ici selon Lindenperg (35) comme l'ensemble des liens formalisés qui se nouent entre les acteurs, sur un territoire, dans une organisation ou institution, pour fédérer les moyens autour de projets ou programmes construits en commun en vue d'atteindre des objectifs partagés. Pour cela la recherche doit combiner et développer de nouvelles postures de recherche, allant de la recherche en «chambre » à la recherche intervention, en passant par la RA.

\section{Innovation}

Ce n'est pas une invention. L'invention c'est la découverte d'un principe, l'action de trouver et son résultat. L'innovation correspond plutôt à l'appropriation de ce principe par le corps social (5). Ce n'est pas un transfert de technique ni un jugement de valeur. Elle n'est pas exogène ou endogène. Son origine peut être interne ou externe, mais en définitive peu importe car en privilégiant l'une ou l'autre, on peut s'interdire de mettre en place des dynamiques exploratoires et de comprendre les processus à l'œuvre. Elle n'est pas linéaire (mais fait des allées et retours, crée des conflits...). Dans ce sens on peut la comparer au tango : il faut de l'imagination, de l'élégance, de l'audace pour le danser et le chemin est fait d'autant de pas en avant que de pas de côté.

L'innovation est un processus sociotechnique et organisationnel de création/destruction, fondé sur la mise en application des savoirs locaux. Dans ce processus les agriculteurs/éleveurs élaborent ou intègrent une ou plusieurs nouvelles techniques dans les systèmes de production au sein des unités de production, pour atteindre des objectifs bien identifiés, individuels et collectifs, et ils construisent le cadre de référence ou «cadre-frontière » (les normes, les règles) à l'intérieur duquel la technique nouvelle va pouvoir s'insérer dans les pratiques socialement reconnues, ce qui confère plusieurs dimensions à l'innovation (technique, sociale, économique, politique...).

L'innovation est socialement construite, c'est-à-dire qu'elle doit être légitimée par un groupe social. Elle est une invention qui a réussi, qui a trouvé son public. En agriculture, le processus est souvent long (plusieurs années) car il remet en cause le cadre de référence ancien, l'ordre établi (routines défensives) et s'appuie sur la déviance. Elle est souvent le fait d'acteurs «quelconques », du moins aux yeux des intervenants ou des observateurs extérieurs (les paysans sont de grands innovateurs...).

\section{Conseil ou service}

Service signifie ici toute activité proposée (matérielle ou immatérielle) aux entreprises ayant pour but d'améliorer les processus de production, de commercialisation ou d'organisation. Ainsi le terme générique de service se réfère aussi bien au conseil/appui (activité typiquement de service), qu'aux activités d'approvisionnements en intrants et en équipements, et d'attributions du crédit, ainsi qu'aux soins.

D’une façon générale, on peut distinguer deux types de service: immatériel et matériel ; marchand ou administré (32). Un service immatériel vise à apporter une information, un conseil, à accompagner un acteur dans un processus de prise de décision (formuler les questions, explorer les solutions et les mettre en place, tirer les leçons), à autonomiser/former les acteurs dans la prise de décisions. Il est possible également, à partir de ce travail d'appui/accompagnement, de capitaliser dans des référentiels technico-économiques nécessaires à la prise de décision. Il se crée alors une relation d'échange plus durable que celle créée par une relation marchande simple. Dans le deuxième cas, matériel, il s'agit d'apporter un produit - au sens de l'économie -, y compris le crédit, pour permettre la réalisation des travaux et organiser les chantiers (production, transformation, commercialisation). Dans ce cas, l'échange ne donne pas nécessairement naissance à une relation stable. Le service immatériel renvoie donc aux processus de choix, à l'élaboration de la stratégie, alors que les services matériels se réfèrent aux moyens à mobiliser pour accomplir les tâches ou activités.

Pour un certain nombre d'auteurs, le conseil est une activité de service immatérielle (31) et intellectuelle, dans le sens ou il ne produit pas de biens physiques. Selon Bounfour (12), il existerait deux façons de concevoir le conseil : pour les uns il correspond à « toute forme d'aide concernant le contenu, le processus ou la structure d'une tâche ou d'une série de tâches », pour les autres « il s'agit d'un service contracté et offert à des organismes par des personnes spécialement formées et qualifiées, qui fournit l'assistance, de manière objective, à l'organisation cliente dans l'identification des problèmes de gestion, analyse des problèmes, recommande des solutions à ces problèmes et aide, lorsqu'on le lui demande, à la mise en œuvre de solutions » (12).

Pourquoi les agriculteurs ont-ils besoin d'un conseil ou d'un service? Les agriculteurs, comme tous les acteurs économiques, ont une rationalité limitée et procédurale (41). Ils sont généralement confrontés à des situations de forte incertitude et doivent décider dans l'urgence. Ils vont donc faire appel au conseil parce qu'ils n'ont pas le temps et/ou parce qu'ils n'ont pas les connaissances pour explorer l'ensemble des possibles et les conséquences de leur 
décision dans un laps de temps suffisamment court pour répondre aux trois questions classiques : que faire ? comment le faire ? pourquoi le faire ?

D'un point de vue de la systémique (figure 1), l'action du conseiller ou du prescripteur va modifier les sous-systèmes de décision et les agriculteurs vont modifier le sous-système opérant à travers le changement des pratiques. En faisant appel au conseil, les agriculteurs essaient donc d'augmenter les champs du possible. Selon Hatchuel (27), une relation de conseil fait référence à trois types de logiques, visant soit la prescription de fait (les états du monde), soit le processus technique, soit la finalité de la décision (jugement), et généralement ces trois logiques sont présentent ensemble à des degrés divers dans une situation de conseil.

- Dans la prescription de fait, le prescripteur comble une incertitude relative à des états du monde, parfaitement connus du demandeur, mais qu'il ne peut constater lui-même. Il s'agit d'une attestation sur la justesse de la chose (garantie). Ceci renvoie, dans le domaine concerné ici, au type d'animal (à la race et aux qualités liées à la race) ou au type de matériel proposé par les forgerons.

- Lorsque la prescription concerne un processus technique, le prescripteur apporte des notions initialement inconnues du demandeur pour éclairer son choix, contribuant ainsi à des modifications des champs d'action et à la réduction des incertitudes notionnelles. Il ne s'agit pas de répondre par oui ou par non, mais de remettre en cause la question initiale, ouvrir les champs du possible, modifier les pratiques et le système de production, etc. Dans le cas présent, c'est l'introduction même de la traction animale dans le système de production et ses conséquences sur les transformations du système de production qui font l'objet de la prescription.

- Dans la prescription de jugement, le prescripteur ne va pas seulement influer sur les aspects techniques (sur la chose), mais il peut également influer sur le jugement de la chose, c'est-à-dire sur les critères qui permettent de justifier le choix, la rentabilité, la qualité, etc. On entend souvent dire que la traction animale permet d'améliorer les conditions de travail ; c'est un constat fait par les agriculteurs eux-mêmes. Mais ils vont chercher dans le conseil ou

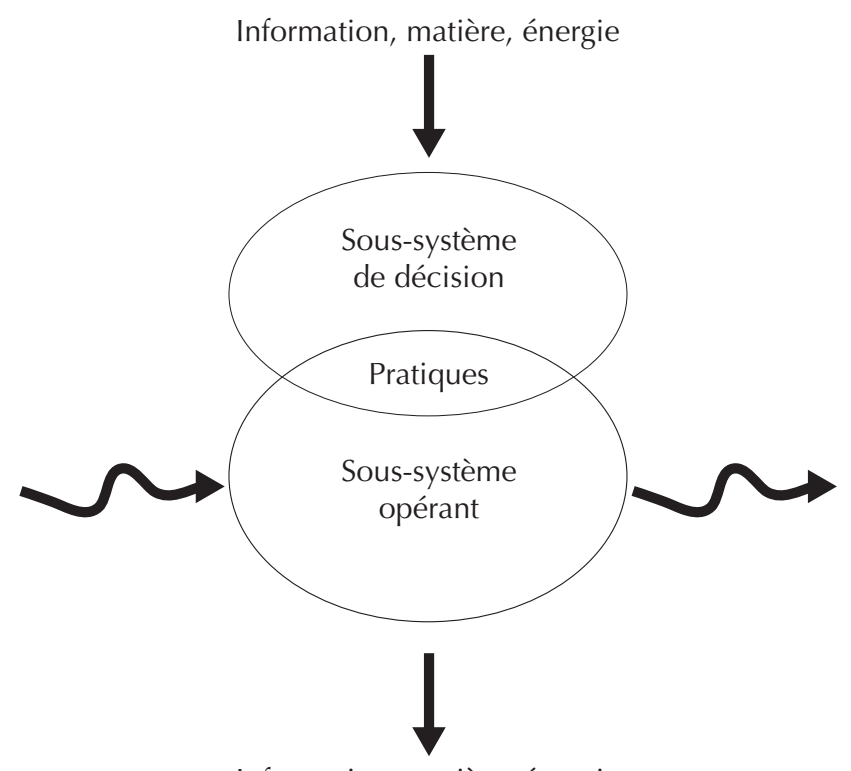

Information, matière, énergie

Figure 1 : schématisation du système de production et des sous-systèmes associés (source: Landais et Deffontaines, 1988, Inra). la prescription un appui pour justifier l'achat d'animaux ou la pratique de la culture attelée.

Selon Hatchuel (27), «Un acteur économique aura d'autant plus recours au conseiller [prescripteur] qu'il se trouve face à un apprentissage par tâtonnement impossible [...], ou bien trop long et trop coûteux. On ne réinvente pas aisément la médecine, nul ne peut essayer tous les restaurants pour choisir où il va aller dîner ». C'est le cas des agriculteurs qui, face à une situation où le risque de pertes est important et la décision non "différable », ne peuvent se permettre de rentrer dans un processus de tâtonnement, d'autant plus que l'étendue de leurs connaissances ne permet pas d'évaluer les risques en toute connaissance de cause. D'où le recours à un conseiller ou prescripteur.

«Ce recours [au conseiller ou prescripteur] s'impose aussi lorsque l'on se trouve en situation que nous qualifierons de 'carence pragmatique', autrement dit lorsqu'il ne s'agit pas de savoir si telle ou telle décision est la meilleure, mais plutôt comment formuler le problème auquel on est confronté et comment juger de sa résolution ; c'est une situation d'indécidabilité, d'angoisse, de crise de la représentation qui découle de l'absence de cadres et de repères d'action. » En agriculture, lorsqu' on travaille au niveau du développement avec une multitude d'acteurs, c'est à ce type de situation que l'on a affaire. Girin (26) utilise dans ce cas la notion de «situation de gestion » pour caractériser les situations où des acteurs décident de travailler ensemble: «nous sommes en présence d'une situation de gestion chaque fois qu'à un ensemble d'activités en interaction est associée l'idée d'activité collective et de résultat faisant l'objet d'un jugement, et que des agents se reconnaissent comme participant à des degrés divers à la production du résultat. » Cela ne signifie pas que toutes les activités élémentaires sont orientées vers la production d'un résultat unique, «il est au contraire possible (et généralement, c'est le cas) que certaines des activités aillent objectivement à son encontre ». Les situations de gestion favorisent l'apprentissage collectif, en particulier lorsqu'il s'agit du conseil et de l'accompagnement (38). Ce sont donc dans ces situations que les outils, démarches, qui favorisent le dialogue entre les acteurs et permettent de se construire une représentation commune, sont nécessaires.

\section{- SITUATIONS DE GESTION, RECHERCHE-ACTION ET PARTENARIAT}

Les problèmes auxquels nous sommes confrontés, lorsque nous travaillons sur le développement rural, les changements, les innovations au niveau des exploitations agricoles, sont, d'une façon générale, complexes, mal définis, mal structurés ou mal posés par des individus qui ne sont pas, nécessairement, des acteurs collectifs ; c'est-à-dire qu'ils ne sont pas reconnus par l'ensemble de la collectivité comme légitimes pour négocier, discuter avec la recherche de ce qu'il convient de faire et comment le faire.

Les recherches en sciences sociales ont, au moins depuis Lewin (34), entrepris d'élaborer et de théoriser des pratiques visant à associer, suivant des modalités variables, les acteurs à la construction et à la conduite de leurs dispositifs d'étude *. Recherche-développement (16), recherche participative (14), recherche clinique (17), recherche-action $(36,37)$ sont autant de termes dont la mise en circulation témoigne de cet effort. Le cadre de travail général d'une recherche-action en partenariat (RAP) est résumé dans le tableau I.

* Cette thématique a ainsi fait l'objet de nombreux débats dans les années 197080, cf. par exemple les numéros spéciaux qui lui ont été consacrés à cette époque dans Connexions en 1977, la Revue internationale d'action communautaire (30), la Revue de l'Institut de Sociologie (6) ou la revue Pour (29). 


\section{Tableau I}

Cadrage et travail à effectuer dans des situations de gestion à partir d'une recherche-action en partenariat

$\begin{array}{lll}\text { Cadrage, } & \text { Travail avec les acteurs } & \text { Travail des chercheurs }\end{array}$

\section{travail à effectuer 1}

- Comprendre la complexité des situations

- Identifier les pratiques et les savoir-faire

- Produire des connaissances sur les phénomènes biochimiques, agronomiques, zootechniques, socioéconomiques et organisationnels

- Produire des connaissances actionnables ${ }^{2}$

- Préciser les conditions de production des connaissances et son domaine de validité

- Produire des méthodes d'intervention

- Produire des connaissances actionnables ${ }^{2}$

${ }^{1}$ La frontière entre analyse et action n'est pas toujours évidente, d'autant plus que dans le cas présent il s'agit de comprendre (analyser) pour agir.

${ }^{2}$ Telles que définies par Argyris et Schön

Lorsque les agriculteurs sont confrontés à des problèmes qui dépassent le cadre de leurs exploitations, comme, par exemple, l'usage collectif de ressources « communes » portées par des acteurs divers et dont les intérêts sur ce plan ne sont pas a priori convergents, on est là face à des situations où se mêlent, selon des configurations localement très variables, des questions d'ordre à la fois technique, économique, social et culturel qui mettent en jeu des (re)définitions de rôle. Il est donc nécessaire, si l'on veut les étudier et contribuer à trouver des solutions, de travailler en équipe pluridisciplinaire, pratique malheureusement peu développée dans les instituts de recherche et les universités. Ceci conduit à élaborer de nouveaux ensembles de pratiques matérielles, de nouvelles formes de relations professionnelles et sociales, et de nouvelles identités collectives; il en découle tout un travail d'ajustement qui nécessite de produire des références nouvelles, des actions inédites, ainsi que la mise en place de nouveaux dispositifs et de nouvelles formes de coopération ou de coordination entre les différents acteurs engagés dans l'aventure (y compris la recherche) (10).

Une telle situation constitue, pour la recherche agronomique, un double défi : i) produire des connaissances scientifiques qui intègrent cette transformation à l'œuvre de ses objets « traditionnels »; et ii) intervenir sur l'objet d'étude pour aider les acteurs à traiter et à résoudre les problèmes que leur pose la situation nouvelle à laquelle ils sont confrontés.

La recherche est donc appelée à imaginer, développer, de nouvelles « postures » d'intervention, dans des situations où ses pratiques et celles des acteurs du développement sont de plus en plus étroitement imbriquées (15).

Aggeri pense que le partenariat (régional) contribue à dépasser les limites des modèles de production de connaissances classiques (laboratoire...), en donnant plus d'initiative et d'autonomie aux acteurs (prévention des crises), et suscite "l'émergence de nouveaux champs de recherche et d'innovation qui aient une certaine pérennité dans le temps » (1). Segretin, citée par Aggeri (1), définit cette situation comme étant un partenariat d'exploration, c'està-dire une situation «qui présente de fortes incertitudes sur les connaissances à mobiliser, sur les objectifs à poursuivre, dans laquelle le cadre de la coopération et les objets du partenariat sont à construire ». Parmi les différentes manières de travailler avec les acteurs de façon partenariale, sera illustrée ici celle qui correspond à la RA.

La recherche-action est née $(19,21,36,37)$ de la rencontre entre une intention de recherche, c'est-à-dire un ensemble d'hypothèses, de méthodes de travail (dispositifs) et des objectifs communs à une communauté de chercheurs, et une volonté de changement exprimée de la part des acteurs de terrain. Le processus de négociation qui s'établit entre ces deux collectifs donne naissance à un cadre éthique partagé $(34,36,37)$. La RA vise donc à la résolution des problèmes et à la création des connaissances sur des phénomènes complexes, où se mêlent phénomènes physiques, biologiques, économiques, sociaux, culturels (croyance et mythe).

Une RA peut se construire soit lorsque c'est l'équipe (ou une partie de l'équipe) qui provoque la RA, soit lorsque les acteurs de terrain (ou usagers de la RA) font appel à l'équipe (ou à une partie de l'équipe). Il est à noter que le terme usager signifie les acteurs autres que les chercheurs et ne doit pas être pris dans le sens ordinaire de celui qui utilise, car il s'agit bien ici de coproduire.

Dans le premier cas, il convient de s'assurer de la légitimité des acteurs pour saisir la recherche, pour formuler le problème à résoudre. Un premier travail consiste en un diagnostic de la situation et du rôle des acteurs dans l'organisation ou la situation. Il se peut qu'à l'issue de ce diagnostic la question soit reformulée et le partenariat complété. C'est à ce moment-là que commence la négociation.

Dans le deuxième cas, il s'agit d'identifier les acteurs, l'organisation, formuler des questions, identifier les préoccupations et/ou les difficultés auxquelles les acteurs sont confrontés et qui peuvent faire l'objet d'un travail de RA.

Cependant, quelle que soit l'origine de la RA elle sera le produit des deux histoires parallèles, celle des acteurs ou usagers qui se traduit par une volonté de changement, et celle de la recherche qui se traduit par une intention de recherche et la nécessité de produire des connaissances (ou du savoir-faire) «actionnables » (dans le sens du terme défini par Argyris et Schön). 
On peut identifie trois phases $(18,21,36,37)$ dans le déroulement d'une recherche-action :

- la phase initiale de mise en route, où à l'issue de laquelle les acteurs auront formalisé, sous forme contractuelle, leurs engagements, leurs devoirs, la structure de fonctionnement, le budget, l'utilisation des données...; ce que Liu appelle le cadre éthique négocié ;

- la phase de réalisation, où il est important d'associer dans les différentes activités les usagers, de fixer le rythme et les modalité d'échanges entre les chercheurs et les usagers, et de développer les moments de restitution des résultats et de régulation des activités ; - la phase de désengagement ou de renégociation, éventuellement, d'une nouvelle RA, d'où l'importance dans la phase initiale de fixer les temps de travail, les délais..

Bien que, dans une RA, la phase de négociation entre chercheurs et usagers joue un rôle essentiel et qu'il soit important, à ce niveau, de déterminer le point de vue des acteurs, engagés ou non dans cette opération, il est également indispensable de réfléchir à l'organisation du déroulement de la recherche pour comprendre la nature du savoir qui est produit $(20,24)$. Le succès ou l'échec d'une RA repose souvent, en effet, sur les conditions dans lesquelles un sujet d'étude a été initialement déterminé, la conception des rôles et statuts des chercheurs, et l'ensemble de l'entreprise impliquée dans la recherche (9). Le contrat, entre le commanditaire et l'équipe de recherche, constitue le point de rencontre entre une demande financière et de terrain de la part du chercheur, et une demande de connaissance et d'action de la part des agents de l'organisation. Le contrat ne lie pas seulement les partenaires sur la forme mais aussi sur le fond, à travers les dates de remise des résultats, les rencontres avec l'ensemble des partenaires, etc. Le contrat conditionne donc la production et le rythme de production des chercheurs $(26,36,37)$.

En ce qui concerne l'équipe de recherche, un premier travail important entre les chercheurs consiste à créer un langage commun entre les différentes disciplines, car parmi les difficultés à surmonter dans un travail pluridisciplinaire et en partenariat, le langage scientifique de l'autre peut être rebutant (43). La mise en place d'un langage commun contribue non seulement à éviter les malentendus, mais surtout à favoriser la construction d'un projet scientifique commun $(20,33)$. Ensuite, il s'agit de construire un corps d'hypothèses, comme l'explique Vinck à propos des travaux en interdisciplinarité : «les chercheurs devraient, ainsi, tout d'abord se mettre d'accord sur le problème à étudier, définir un projet ou une visée commune, s'accorder sur la manière d'analyser le problème, élaborer des concepts communs et construire un langage partagé, les concepts devraient être explicités, de même que les hypothèses de base. » La RA utilise aussi des objets intermédiaires. Il s'agit «des objets produits ou utilisés au cours du processus de conception, traces et supports de l'action de concevoir, en relation avec les outils, procédures, et acteurs ». La capacité d' «analyseurs » des objets intermédiaires dans des processus de conception est due à leur caractère hybride : "ils sont relatifs à la fois à la mise en forme de la nature d'un produit nouveau et à l'organisation de la coordination entre ses concepteurs. »

Ainsi les cartes, mais aussi les typologies d'exploitation, des territoires, etc., en tant que modèle, lorsqu'elles sont utilisées pour identifier, délimiter des phénomènes dans les territoires, sont à considérer comme des objets intermédiaires. L'auteur a utilisé, dans des RA, la programmation linéaire comme un objet intermédiaire pour élaborer une représentation commune du fonctionnement des exploitations agricoles entre chercheurs (de disciplines différentes) et aussi pour dialoguer avec les agriculteurs. En effet, l'auteur a pu dialoguer avec les agriculteurs sur les conséquences techniques des propositions et ensemble ils se sont mis d'accord sur les scénarios d'évolution à tester.
Trois autres éléments sont importants dans la réussite d'une RA, et cela de la phase initiale ou de mise en place; il s'agit de la capacité à enrôler des acteurs (du développement, de la recherche...), à faire des alliances (avec l'administration, les hommes politiques, la recherche...), à identifier les porte-parole, et à traduire les logiques (celle des agriculteurs, des conseillers, des chercheurs, du chef du village...) et les rationalités individuelles dans un projet qui ait un sens pour la majorité des acteurs engagés dans la RA (13).

Cette attitude « ouverte » comporte ses propres limites, car le chercheur est confronté à la nécessité de produire de la connaissance, de restituer des observations, d'établir des modèles. Le passage de l'observation à l'interprétation et à la construction théorique (caractéristique d'une démarche de recherche) ne peut s'opérer sans privilégier à un moment donné certains faits, sans les ordonner et les schématiser, et sans s'appuyer sur des intuitions normatives. La résolution de cette dialectique entre objectivation et herméneutique (9) est au cœur de la RA. Elle ne résulte pas simplement d'une partie ou d'un comportement mais est très liée à la méthodologie et au dispositif formel de la recherche $(22,23)$. Les phases de négociation et de pré-étude permettent justement de définir et d'instituer ce dispositif de relation.

Ci-après sont présentés les différents comités qui permettent d'assurer la gouvernance d'une RA (l'auteur s'appuie ici sur une expérience à laquelle il a participé pour illustrer l'importance de certains comités). On peut distinguer les comités du début du programme qui correspondent à un besoin soit de négociation, soit d'arbitrage, soit de gestion et d'amélioration du fonctionnement de la recherche, et les comités qui sont parfois mis en place, au fur et à mesure de l'évolution de la recherche, en fonction de l'évolution de la situations et des nouveaux besoins ou problèmes.

\section{Comité d'animation de la recherche-action}

Ce comité comprend des collègues ayant la responsabilité de l'animation des équipes de travail. Sa fonction consiste à orienter les actions de recherche, à créer de nouvelles actions, à fixer des priorités dans le travail, à organiser et enfin à conduire les négociations (tout au long de la recherche), ainsi que la préparation du rapport final et, si besoin est, de renégocier une nouvelle recherche-action. Ce groupe doit également :

- élaborer des modèles ou théories communes (40) ; la formalisation du problème par les chercheurs passe par l'élaboration d'un langage commun, par un effort de conceptualisation interdisciplinaire et par la modélisation des phénomènes complexes ;

- préparer les restitutions entre les acteurs, mettre en commun des représentations de la situation, construites par les chercheurs et validées par l'ensemble des acteurs, et élaborer de nouvelles hypothèses de travail, ainsi que la mise en place de nouveaux dispositifs de recherche.

Ce comité se heurte à différentes difficultés, comme la disponibilité des membres de l'équipe. Mais le caractère pluridisciplinaire de ce comité crée les conditions idéales d'une homogénéisation du langage entre les différentes disciplines.

\section{Comité de gestion de la recherche-action}

Ce comité, généralement prévu dans le contrat de recherche, est constitué par les organismes finançant le programme, certains membres de l'équipe de recherche, ainsi que des représentants de la profession agricole aux niveaux local et régional et des personnes ressources locales. Il ne s'agit pas de faire un miniparlement, mais plutôt de le faire le plus représentatif possible. Il a pour objectif d'éviter des conflits et un empiètement dans le travail d'animation et de développement. Il doit veiller au bon déroulement de l'opération en facilitant les rencontres entre les différents acteurs et en signalant 
les dysfonctionnements. Il avalise les nouvelles actions en discutant les acquis. Dans le cas de la recherche Vittel, ce comité n'a pas joué le rôle prévu à l'origine. Il a dû traiter les relations politiques entre l'entreprise et les représentants des agriculteurs, et entre ces derniers et l'équipe de recherche.

\section{Comité scientifique de pilotage de la recherche-action}

Ce comité a un rôle d'orientation scientifique. Composé par des scientifiques reconnus dans les diverses disciplines concernées et dans ce type de recherche, il préserve l'équilibre entre la production des connaissances scientifiques et la résolution des problèmes (production des connaissances actionnables). C'est à l'occasion de la présentation de l'avancement des travaux dans ce comité que les chercheurs peuvent prendre du recul par rapport au terrain et élaborer de nouvelles connaissances scientifiques.

Dans le cas de la RA à Vittel, cette organisation, en comités et équipes, a été extrêmement lourde ; aussi peut-on s'interroger sur la possibilité de procédures moins exigeantes. Il est difficile de valider et de justifier les diverses structures de négociation mises en place, mais il apparaît qu'elles correspondent à la complexité du problème posé et à la nécessaire participation des acteurs. Tout l' «art» a été pour l'auteur de garder en permanence l'équilibre « négocié » entre l'action et la recherche. Par ailleurs, une démarche de RA est dynamique et suppose un certain degré de souplesse ou de flexibilité, en particulier au niveau du dispositif.

Ainsi les différents comités ont favorisé la création d'un langage commun aux divers acteurs ; des représentations communes ont servi à la fois de moyen de communication, de source de production scientifique et de lieu de formation des différents acteurs. Le comité d'animation de la recherche a été un lieu où de nouvelles actions interdisciplinaires ont pu être discutées et élaborées, par exemple la création d'une base de données commune à l'ensemble des disciplines, avec l'objectif d'étudier les aspects territoriaux des changements des systèmes de production. Le dispositif de RA est un dispositif dynamique et évolutif, comme le montre la figure 2. En effet, l'imprévu fait partie d'une RA, que ce soit au niveau des questions à traiter ou dans d'autres aspects; il est donc nécessaire de former de nouvelles équipes ou de recomposer les anciennes. Que de tensions ou de conflits entre les acteurs qu'il faut être capable de gérer ! Ce fut le cas pour l'auteur avec le comité politique.
VERS QUELLE RECHERCHE

\section{EN PARTENARIAT POUR LE DEVELOPPEMENT}

DE LA TRACTION ANIMALE?

Lors de l'atelier où ce document a été présenté, nombreux ont été les intervenants qui se sont demandés comment étendre l'utilisation de la traction animale au plus grand nombre d'agriculteurs intéressés par la technique et comment rendre la technique plus durable. Pirot et coll. (39) affirment que la recherche en partenariat constitue la principale voie pour accroitre non seulement le nombre de paysans utilisateurs de la technique mais également pour coproduire de nouvelles innovations, sociotechniques et organisationnelles, nécessaires au développement durable de la technique et des systèmes de production.

Il est à noter également que les différentes présentations faites à l'atelier ont aussi mis en évidence l'importance d'autres acteurs de la filière (forgerons, conseillers, vétérinaires, techniciens vétérinaires) dans l'utilisation de la traction animale, sans oublier les conditions économiques (existence des marchés des produits et des animaux) et financières (crédit) qui favorisent son adoption. On peut aussi ajouter, à ce rapide tour d'horizon, la faible organisation des acteurs, tant au niveau des agriculteurs qu'à celui des forgerons ou des vétérinaires, ce qui semble être le principal frein au développement de la traction animale.

La question est donc : comment dans une telle situation (diversité des acteurs, des logiques et des stratégies) mettre en place une recherche en partenariat et coconcevoir des innovations sociotechniques et organisationnelles?

Quelques pistes sont esquissées ci-après par l'auteur qui s'est appuyé sur les résultats de l'atelier et sur l'article de Pirot et coll. (39) qui traitent des questions d'innovation avec une composante traction animale. On pourrait choisir avec une ou plusieurs organisations professionnelles, ONG, des services de l'Etat, un échantillon de plusieurs localités où la question de la traction animale (utilisation) ou de son fonctionnement et de sa pérennité (manque de services) est posée : i) identifier *, à travers un diagnostic, les acteurs impliqués, leurs pratiques et stratégies et les questions

* Il s'agit ici typiquement du cas où c'est la recherche qui est à l'origine du processus ou de la demande, et non les acteurs.
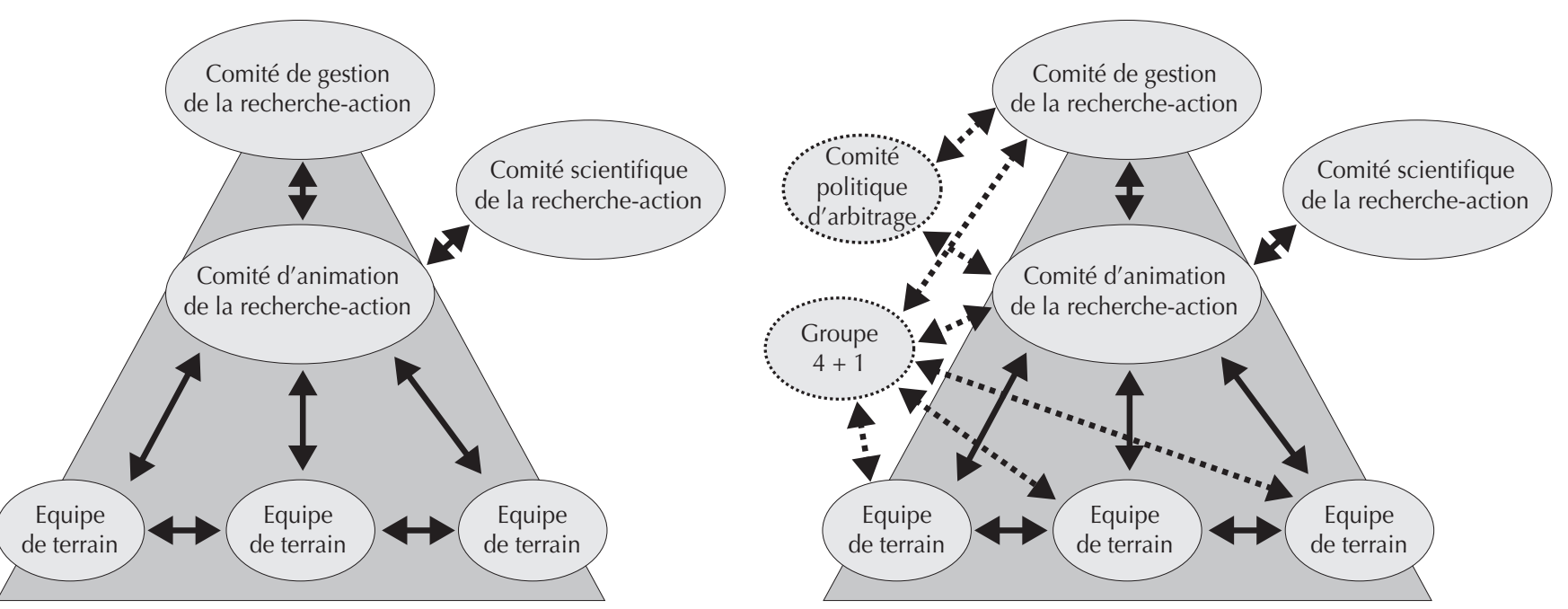

Figure 2 : structure fonctionnelle de la recherche-action : cas de Vittel. 
qu'ils se posent à propos de la traction animale ; ii) sélectionner, lors de la restitution du diagnostic, avec leur participation (traduction de la demande sociale faisant émerger la volonté de changement), des questions traitables par la recherche, c'est-à-dire « traduite » sous forme de question scientifique et de problématique structurante, ce qui intéresse un nombre important de chercheurs (intention de recherche) ; et iii) mettre en place un dispositif de terrain avec l'objectif de formaliser le contrat (cadre éthique) entre la recherche (équipe pluridisciplinaire et pluri-institutionnelle) et l'ensemble des acteurs concernés. Les chercheurs doivent, dans la phase de négociation, développer plus particulièrement leurs capacités de "traducteurs » et de "médiateur», et s'appuyer sur un comité de pilotage de la RA qui se met en place, où des représentants des groupements villageois (paysans, agroéleveurs, éleveurs, forgerons, aides-vétérinaires, conseillers, agents de services...) se rencontrent régulièrement pour formaliser la recherche-action et le partenariat. La réussite ou l'échec de la RA dépendra en grande partie de la négociation initiale, de la qualité des accords et de l'adhésion de l'ensemble des acteurs au projet.

Cependant, dans le cas de la traction animale, la dimension financière de l'investissement ne doit pas être négligée, car plusieurs innovations sociotechniques et organisationnelles n'ont pas résisté, malgré leur bien-fondé, à cause des problèmes financiers.

\section{Remerciements}

Je remercie E. Vall non seulement pour la lecture attentive qu'il a faite d'une première version, mais surtout pour les nombreuses propositions d'améliorations qu'il m'a suggérées. Je remercie également $\mathrm{C}$. Rawski pour le temps qu'elle a consacré à une meilleure lisibilité de cet article. Je tiens aussi à remercier chaleureusement J.-P. Pichot.

\section{BIBLIOGRAPHIE}

1. AGGERI F., 2002. La construction des objets de recherche dans les partenariats d'exploration. In: Symposium Recherche pour et sur le développement régional, Montpellier, France, Inra, 12-13 décembre 2001.

2. AGGERI F., HATCHUEL A., 2003. Ordres socio-économiques et polarisation de la recherche dans l'agriculture: pour une critique des rapports sciences/société. Soc. Trav., 45 : 113-133.

3. AKRICH M., CALLON M., LATOUR B., 1988. A quoi tient le succès des innovations. Ann. Mines, Sér. Gérer Comprendre, 11 : 4-18.

4. AKRICH M., CALLON M., LATOUR B., 1988. L'art de l'intéressement. Ann. Mines, Sér. Gérer Comprendre, 12 : 14-29.

5. ALTER N., 2000. L'innovation ordinaire. Paris, France, PUF, 278 p.

6. A propos de la recherche-action, 1981. Revue Inst. Sociol. (Bruxelles, Belgique), 3.

7. AVENIER M.J., 1992. Recherche-action et épistémologies constructivistes, modélisation systémique et organisations socioéconomiques complexes : quelques «boucles étrangers" fécondes. Revue int. Syst., $6: 403-420$.

8. AVENIER M.J., éd., 2000. Ingénierie des pratiques collectives. Paris, France, L'Harmattan, 411 p. (Coll. Ingenium)

9. BENHHOZI P.J., 1990. La négociation d'une recherche : une étape clé dans la méthodologie d'intervention. Econom. Soc., Sér. Sci. Gestion, 15 : 195-209.

10. BERRY M., 1991. L'interdisciplinarité : un objectif difficile à concrétiser. La recherche, 228 : 66-68.

11. BOSC P.-M., HANAK FREUD E., 1995. Recherche agricole et innovation en Afrique tropicale. Montpellier, France, Cirad, 146 p. (Coll. Repères)
12. BOUNFOUR A., 1989. Vers I'industrialisation du conseil. Revue fr. Gestion, $76: 23-34$

13. CALLON M., 1986. Eléments pour une sociologie de la traduction. La domestication des coquilles Saint-Jacques et des marins-pêcheurs dans la baie de Saint-Brieuc. L'année sociol., 36 :169- 208.

14. CHAMBERS R., 1992. Rural appraisal: rapid, relaxed, and participatory. Discussion paper 311. Brighton, UK, University of Sussex, Institute of Development Studies.

15. CHANAL V., LESCA H., MARTINET A.C., 1997. Vers une ingénierie de la recherche en sciences de gestion. Revue fr. Gestion, 116 : 41-51.

16. CHAUVEAU J.P., CORMIER-SALEM M.C., MOLLARD E., éds, 1999. L'innovation en agriculture. Questions de méthodes et terrains d'observation. Paris, France, IRD, 362 p. (Coll. A travers champs)

17. CHIA E., 1992. La «recherche-clinique»: proposition méthodologique dans I'analyse des pratiques économiques des agriculteurs (étude de cas en Lorraine). Paris, France, Inra, 39 p. (Coll. Etudes et recherches sur les systèmes agraires et le développement $n^{\circ} 26$ )

18. CHIA E., BARBIER M., 1999 Gestion et qualité de l'eau : apprentissage collectif et rôle des prescripteurs. Cah. Agric., 8 : 109-117.

19. CHIA E., BROSSIER J., BENOIT M., 1992. Recherche-action : qualité de I'eau et changements des pratiques agricoles. Econ. rurale, 208-209 : 30-36.

20. CHIA E., DEFFONTAINES J.P., 1999. Pratiques et dispositifs de recherche face à un problème d'environnement. Nat. Sci. Soc., 7 : 31-41.

21. CHIA E., RAULET N., 1993, Agriculture et qualité de l'eau : négociation et rôle de la recherche, le cas du programme Agrev. In : Cerf M., éd., Qualité et systèmes agraires, techniques, lieux, acteurs. Paris, France, Inra, p. 177-193. (Coll. Etudes et recherches sur les systèmes agraires et le développement $\mathrm{n}^{\circ} 28$ )

22. DAVID A., 2001. La recherche-intervention, cadre général pour la recherche en management? In : David A., Hatchuel A., Laufer R., éds, Les nouvelles fondations des sciences de gestion. Paris, France, Fnege, p. 193-202.

23. DAVID A., HATCHUEL A., LAUFER R., éds, 2001. Les nouvelles fondations des sciences de gestion. Paris, France, Fnege, 213 p.

24. DEFFONTAINES J.P., CHIA E., 1992. Une recherche-action sur un système agraire soumis à des impératifs de qualité de l'eau. Résultats et bilan méthodologique. C.R. Acad. Agric. fr., 78 : 65-78.

25. FLICHY P., 1995. L'innovation technique : récents développements en sciences sociales. Vers une nouvelle théorie de l'innovation. Paris, France, La Découverte, 207 p.

26. GIRIN J., 1990, L'analyse empirique des situations des situations de gestion, éléments de théorie et de méthode. In : Martinet A.C., éd. Epistémologie et sciences de gestion. Paris, France, Economica, p. 141-182.

27. HATCHUEL A., 1995. Les marchés à prescripteurs. Crises de l'échange et genèse sociale. In : Jacob A., Vérin H., éds, L'inscription sociale du marché, Colloque de l'Association pour le développement de la socio-économie, Lyon, France, novembre 1992. Paris, France, L'Harmattan, 296 p.

28. LANDAIS E., DEFFONTAINES J.P., 1988. Les pratiques des agriculteurs. Point de vue sur un courant nouveau de recherche agronomique. Paris, France, Inra, p. 125-158. (Coll. Etudes et recherches sur les systèmes agraires et le développement $n^{\circ} 109$ )

29. La recherche-action, 1983. Pour (Toulouse, France), 90.

30. La recherche-action, enjeux et pratiques, 1981. Revue int. Action communautaire (Montréal, Canada), 5/45.

31. LAURENT C., CERF M., PASQUIER C., 2002. Le conseil en agriculture : un investissement immatériel entre développement sectoriel et développement territoriale. Géograph., Econ. Soc., 4 : 131-153.

32. LAVILLE J.L., 2005. Sociologie des services. Entre marché et solidarité. Paris, France, Eres, 180 p.

33. LEMERY B., BARBIER M., CHIA E., 1997. La recherche-action en pratique. Réflexions autour d'une étude de cas. In: Albaladejo C., Casabianca F., éds, La recherche action. Ambitions, pratiques, débats. Paris, France, Inra, p. 71-89. (Coll. Etudes et recherches sur les systèmes agraires et le développement $\mathrm{n}^{\circ} 30$ )

34. LEWIN K., 1959. Psychologie dynamique. Paris, France, PUF, 296 p. 35. LINDENPERG G., 1999. Les acteurs de la formation professionnelle pour une nouvelle donne. Rapport au Premier ministre. Saint-Denis-La Plaine, France, Centre Inffo, 135 p. (www.centre-inffo.fr) 
36. LIU M., 1992. Présentation de la recherche-action : définition, déroulement et résultats. Revue int. Syst., 6 : 293-311.

37. LIU M., 1992. Vers une épistémologie de la recherche-action. Revue int. Syst., $6:$ : 435-454.

38. MAXIME F., CERF M., 2002. Apprendre avec I'autre: le cas de I'apprentissage d'une relation de conseil. Educ. perm., $151:$ 47-68.

39. PIROT R., HAVARD M., VALL E., KEMTSOP G.A., FALL A., 2004. Conditions d'émergence et de pérennisation des services d'appui à la traction animale en Afrique subsaharienne : cas des agroéquipements. In Actes atelier Traction animale et stratégies d'acteurs: quelle recherche, quels services face au désengagement de l'Etat ? Bobo-Dioulasso, Burkina Faso, 17-21 novembre 2003. Revue Elev. Méd. vét. Pays trop., 57 (3-4).

\section{Summary}

Chia E. Principles, Methods of Participatory Research: Proposal for Draft Animal Power

The meeting of researchers, who question themselves on the efficiency of their actions when they accompany stakeholders during change processes, provides the opportunity to ponder on the research methods to develop when working together with the stakeholders: participative research, research-action, research-intervention... The author proposes to present the research-action approach as new. If the three phases of research-action are important, the negotiation phase is essential, because it enables contract formalization among partners (ethical aspect), development of a common language, and formalization of structuring efforts between researchers with various specialties and stakeholders. In the research-action approach, the managing set-ups (scientific committees...) play a major role: they guarantee at the same time a solution to problems, production, and the legitimacy of the scientific knowledge produced. In conclusion, the author suggests ways to develop research-action in the field of animal traction in order to conceive new socio-technical and organizational innovations that will make the use of this technique easier.

Keywords: Animal power - Research - Partnership - Innova-
40. SEBILLOTTE M., 2001. Des recherches en partenariat "pour » et "sur » le développement régional. Ambitions et questions. Nat. Sci. Soc., 9 : $5-7$.

41. SIMON H.A., 1978. Rationality as process and as a product of thought. Am. econ. Rev., $68: 1-16$.

42. VALL E., LHOSTE P., ABAKAR O., DONGMO NGOUTSOP A.L., 2003. La traction animale dans le contexte en mutation de l'Afrique subsaharienne : enjeux de développement et de recherche. Cah. Agric., $12: 219-226$.

43. VINCK D., 2003. L'instrumentation du travail interdisciplinaire : cadrage des échanges et médiation par les objets intermédiaires. Esprit crit., 5. (www.espritcritique.org)

\section{Resumen}

Chia E. Principios, métodos de la investigación en asociación : una propuesta para la tracción animal

El encuentro de los investigadores que se preguntan sobre la eficacia de sus actos para acompañar a los protagonistas en los procesos de cambio, representa una ocasión para interrogarse sobre los métodos de investigación a desarrollar durante los trabajos realizados con los protagonistas: investigación participativa, investigación-acción, investigación-intervención... $\mathrm{El}$ autor propone presentar los pasos a seguir en la investigación-acción como novedad. Si las tres fases de la investigación-acción son importantes, la fase de la negociación es capital, porque permite la formalización del contrato entre los socios (marco ético), la fabricación de un lenguaje común, y la formalización de una problemática estructurante entre los investigadores de las diversas disciplinas y los protagonistas. En el proceso de la investigación-acción, los dispositivos de gobierno (comité científico...) desempeñan un papel muy significativo: son a la vez garantes de solución de los problemas y de la producción, así como de la legitimación de los conocimientos científicos producidos. En conclusión se proponen las pistas para construir la investigación-acción en el campo del transporte animal, para imaginar nuevas innovaciones sociotécnicas y de organización, con el fin de facilitar el uso de esta técnica.

Palabras clave: Energía animal - Investigación - Coparticipación - Innovación - Gestión - Africa al sur del Sahara. 